\title{
MENSURAÇÃO E ANÁLISE DE DESEMPENHO LOGÍSTICO EM SISTEMA DE ESTOQUE GERENCIADO PELO FORNECEDOR E CADEIA DE SUPRIMENTOS EM TRÊS NÍVEIS
}

\author{
Thiago André Guimarães \\ thiandgui@gmail.com \\ Centro Universitário Franciscano do Paraná \\ Programa de Pós Graduação em Métodos Numéricos em Engenharia - UFPR \\ Grupo de Tecnologia Aplicada à Otimização - UFPR \\ Cassius Tadeu Scarpin \\ cassiusts@gmail.com \\ Programa de Pós Graduação em Métodos Numéricos em Engenharia - UFPR \\ Grupo de Tecnologia Aplicada à Otimização - UFPR \\ Maria Teresinha Arns Steiner \\ maria.steiner@pucpr.br \\ Programa de Pós Graduação em Engenharia de Produção e Sistemas - PUCPR
}

\begin{abstract}
Resumo
Este artigo destina-se à mensuração de desempenho de problemas integrados de roteirização e estoque em uma cadeia de suprimentos com três níveis, consistindo de uma base, um fornecedor (depósito) e um conjunto de clientes dispersos geograficamente. Neste cenário, os clientes possuem uma demanda estocástica normalmente distribuída, conhecida a priori, e uma capacidade de armazenamento limitada. O fornecedor gerencia os níveis de estoque dos clientes e decide quando, em que quantidade e de que forma abastecê-los, a fim de que não ocorram rupturas ao longo de um horizonte de tempo finito. Para tal, uma frota limitada de veículos de mesma capacidade está disponível no depósito. Cabe também ao fornecedor decidir sobre seu próprio nível de estoque e solicitar a reposição deste a partir de uma base distante. O objetivo global da operação é minimizar os custos logísticos envolvidos. Para tal, o estudo avalia comparativamente duas diferentes políticas de distribuição a partir de um conjunto de indicadores logísticos sistêmicos, envolvendo métricas de transporte e estocagem. Em virtude da característica fortemente combinatória do problema, uma estratégia heurística é proposta para sua resolução. Experimentos computacionais realizados sob diferentes cenários permitiram observar um limitante para ganhos de escala no custo de transporte, além de um padrão constante de estocagem dos clientes independentemente da política de distribuição adotada.
\end{abstract}

Palavras-chave: Análise de Desempenho, Roteirização e Estoques, Cadeia em Três Níveis.

\begin{abstract}
This paper address to performance measurement on integrated routing and inventory problems in a supply chain with three echelons, consisting of a supply base, a supplier (depot) and a set of geographically dispersed customers. In this scenario, customers have a stochastic demand, known a priori, and a limited inventory capacity. The supplier manages customer inventory levels and decide when, how much and how to combine them into a route, in order to avoid stock out, over a finite time horizon. For this, a limited fleet of vehicles with same capacity is available at the supplier. Suppliers also manage its own inventory level and request replenishment from a distant supply base. The overall objective of the operation is to minimize logistics costs involved. In this context, this paper compares two different distribution policy considering a set of indicators systemic logistics, involving transport and inventory of metrics. Due to the strong combinatorial characteristic of the problem, a heuristic strategy is proposed for its resolution. Computational experiments performed under different scenarios allowed to observe a limiting for scale savings in transportation costs and a constant pattern of customer inventory regardless of the distribution policy adopted.
\end{abstract}

Keywords: Performance Measurement, Inventory Routing, Three-echelon System. 


\section{INTRODUÇÃO}

Sistemas de gerenciamento de estoque pelo fornecedor (VMI - Vendor Managed Inventory) são amplamente reconhecidos pela sua capacidade de criação de valor, tanto para os clientes, que deixam de demandar esforços no controle de seus estoques, quanto para os fornecedores, pela possibilidade de maior coordenação das entregas entre os diferentes pontos de demanda [1]. Entretanto, operacionalização de sistemas VMI requer a resolução de um complexo problema de otimização combinatória, denominado Problema de Roteirização e Estoques (PRE), que consiste na decisão de um fornecedor sobre quando, quanto, e em qual rota abastecer um conjunto de clientes ao longo de um horizonte de planejamento.

Nos últimos trinta anos o PRE vem recebendo ampla atenção da literatura acadêmica, incorporando características mais aderidas às situações reais, como transbordo [2], janelas de tempo [3] e consistência [4]. Ao mesmo tempo, diversas aplicações vem sendo consideradas, entre elas a distribuição de gás natural em ambientes urbanos [5], [6] e marítimos [7], transporte de sangue e plasma entre hospitais [8], entrega de cimento a granel [9], logística de componentes da indústria automobilística [10], [11] entre outras. Ainda assim, abordagens que contemplam cadeias de suprimentos em três níveis são escassas, sendo de conhecimento dos autores apenas os estudos [12], [13]. Apesar da maior dificuldade de resolução, esta variante do IRP incorpora uma decisão importante acerca da reposição do próprio fornecedor. Tal consideração possibilita um tratamento mais condizente à realidade, além de garantir soluções integradas de menor custo [13]. Ao mesmo tempo, um número muito reduzido de pesquisas se dedicam à mensuração e análise de desempenho logístico no âmbito do PRE, limitado à pouco trabalhos como em [14], [15]. Até o melhor conhecimento dos autores, a literatura acadêmica ainda não aborda os dois temas simultaneamente.

Diante de tal lacuna, este trabalho propõe e avalia um conjunto de indicadores sistêmicos para medição e análise de desempenho logístico em PRE com três níveis. Para tanto, duas diferentes políticas de distribuição foram consideradas: maximum level $(M L)$ e order-up to level $(O U)$. Sob a política $M L$, o fornecedor decide livremente quanto entregar ao cliente, desde que sua capacidade de estocagem seja respeitada. Já a política $O U$ implica em abastecer o cliente até sua capacidade máxima de estoque, sempre que ocorrer uma entrega A mensuração e análise de desempenho logístico das diferentes políticas de distribuição possibilitam tratar uma maior variabilidade de situações acerca dos indicadores propostos. Neste sentido, a principal contribuição do trabalho advém dos padrões identificados pelos experimentos computacionais realizados.

O restante do artigo está estruturado como segue: na seção 2 o PRE em três níveis é formalmente definido e um modelo de programação linear inteiro misto binário é proposto. A seção 3 apresenta a sistemática dos indicadores de desempenho logístico Na seção seguinte, a heurística proposta é detalhada. Na seção 5, as instâncias geradas e os resultados obtidos são discutidos e a seção 6 tece as conclusões do trabalho.

\section{PROBLEMA DE ROTEIRIZAÇÃO E ESTOQUES EM TRÊS NÍVEIS}

O PRE em três níveis é formulado sobre um grafo completo e não orientado $G=(V, A)$ onde $V=\{0, \ldots, n+1\}$ é o conjunto de vértices e $\mathrm{A}=\{(i, j) \epsilon V, i \neq j\}$ o conjunto de arcos. O vértice " 0 " refere-se ao fornecedor, o subconjunto $V$ ' $=\{1, \ldots, n\}$ representa os clientes e " $n+1$ " a base. Cada arco (i, j) $\in \mathrm{V}$, possui um custo não negativo $c_{i j}$ e as decisões são definidas em horizonte temporal finito $T=\{0, \ldots, p\}$. A distância entre o fornecedor (vértice 0 ) e a base (vértice $n+1$ ) é dada por $c_{0 n+1}$. O cliente i possui uma demanda $d_{i}^{t}, \forall t \in T$, normalmente distribuída e conhecida a priori, variando ao longo de $T$. Seu custo de estocagem respectivo é dado por $h_{i}$ e sua capacidade de armazenamento $C_{i}$, sendo $m C_{i}$ a quantidade mínima que deve ser mantida no estoque, calculada

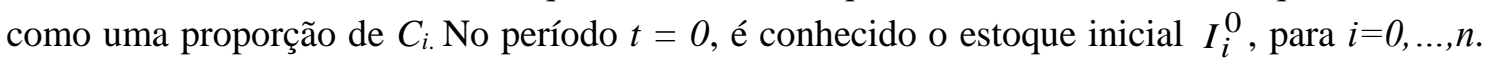
Assume-se que a base possui uma quantidade de produto suficiente para atender à demanda do fornecedor ao $\operatorname{logo}$ de $T$. Este por sua vez, não possui restrição máxima estocagem.

Uma frota homogênea de $K$ veículos, com capacidade de carreamento $L$, está disponível no fornecedor. Cada veículo $k$ pode realizar até uma rota em cada período $t$. Conforme ilustrado na figura 1 e baseado na proposta de [12], o processo de distribuição implica em decidir quando 
abastecer o fornecedor (F) a partir da base (B), quando abastecer o conjunto de clientes $V^{\prime}$ a partir do fornecedor $(\mathrm{F})$ e qual roteiro percorrer para realizar as entregas. $\mathrm{O}$ reabastecimento do fornecedor é responsabilidade da base não utiliza a frota de veículos $K$. A base por sua vez não pode abastecer os clientes diretamente.

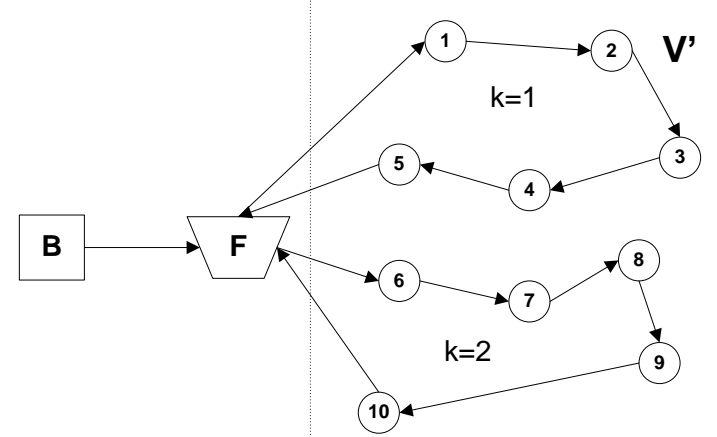

Figura 1. Sistema logístico em três níveis. Fonte: Adaptado de [12]

As restrições do problema são detalhadas a seguir: o estoque do cliente $i$ não deve exceder sua capacidade $C_{i}$ nem ser inferior ao mínimo requerido $m C_{i}$ ao longo de $T$ e o estoque do fornecedor não pode ser negativo em $T$. Cada cliente $i$ deve ser atendido por um único veículo. $\mathrm{O}$ volume transportado pelo veículo $k$ no período $\mathrm{t}$ não deve exceder sua capacidade $L$ e cada veículo deve realizar apenas uma rota de entrega por período, devendo esta iniciar e terminar no depósito. A quantidade de rotas não deve exceder a disponibilidade de veículos da frota.

Inicialmente, para a política de distribuição flexível $M L$, o PRE em três níveis é definido como um modelo de programação linear inteira mista binária (PLIMB) como segue:

$$
\begin{array}{lr}
\text { Minimizar }: \sum_{t=1}^{p} I_{0}^{t} h_{0}+2 \sum_{t=1}^{p} \lambda^{t} c_{0 n+1}+\sum_{i=1}^{n} \sum_{t=1}^{p} I_{i}^{t} h_{i}+\sum_{i=0}^{n} \sum_{j=0, j \neq i}^{n} \sum_{k=1}^{K} \sum_{t=1}^{p} c_{i j} x_{i j k}^{t} \\
m C_{i} \leq I_{i}^{t} \leq C_{i} & \forall t \in T, i \in V^{\prime} \\
q_{i k}^{t} \leq C_{i}-I_{i}^{t-1} & \forall k \in K, t \in T, i \in V^{\prime} \\
I_{i}^{t}=I_{i}^{t-1}+\sum_{k=1}^{K} q_{i k}^{t}-d_{i}^{t}+m C_{i} & \forall t \in T, i \in V^{\prime} \\
0 \leq I_{0}^{t}=I_{0}^{t-1}+r^{t}-\sum_{i=1}^{n} \sum_{k=1}^{K} q_{i k}^{t} & \forall t \in T \\
\sum_{i=0}^{n} q_{i k}^{t} \leq L y_{i k}^{t} & \forall k \in K, t \in T \\
\sum_{k=1}^{K} y_{i k}^{t}=1 & \forall i \in V^{\prime}, t \in T \\
\sum_{i=1}^{n} x_{i j k}^{t}=1 & \forall k \in K, t \in T, j \in V^{\prime} \\
\sum_{i=1}^{n} x_{j i k}^{t}=1 & \\
\sum_{i=1}^{n} x_{0 i k}^{t}=\sum_{i=1}^{n} x_{i 0 k}^{t} & \forall k \in K, t \in T, j \in V^{\prime} \\
\sum_{k=1}^{K} y_{0 k}^{t} \leq K & \forall k \in K, t \in T \\
& \\
& \\
& \\
& \\
& \\
& \\
& \\
& \\
&
\end{array}
$$




$$
\begin{array}{lr}
\sum_{i=0}^{n} x_{i j k}^{t}+\sum_{i=0}^{n} x_{j i k}^{t}=2 y_{i k}^{t} & \forall k \in K, t \in T \\
\sum_{j=0 k=1}^{n} \sum_{j i k}^{K} f_{j=0}^{t}-\sum_{j=1}^{n} \sum_{i j k}^{K}=\sum_{k=1}^{K} q_{i k}^{t} y_{i k}^{t} & \forall t \in T, i \in V^{\prime} \\
f_{i j k}^{t} \leq L y_{i k}^{t} & \forall k \in K, t \in T, i \in V, j \in V^{\prime} \\
f_{i j k}^{t} \geq 0, I_{i}^{t} \geq 0, q_{i k}^{t} \geq 0, d_{i}^{t} \geq 0, r^{t} \geq 0 & \forall k \in K, t \in T, i \in V, j \in V^{\prime} \\
x_{i j k}^{t}, y_{i k}^{t}, \lambda^{t} \in\{0,1\} & \forall k \in K, t \in T, i \in V, j \in V
\end{array}
$$

A expressão (1) minimiza a função objetivo do problema, contemplando o custo de armazenagem e abastecimento do fornecedor, o custo de armazenagem e distribuição dos clientes. $\mathrm{O}$ conjunto de inequações (2) limita o estoque do cliente $i$ em cada período $t$ entre um valor mínimo $m C_{i}$ e um valor máximo $C_{i}$. Em (3) garante-se que a quantidade entregue ao cliente $i$ no período $t$ não exceda sua capacidade disponível. As equações em (4) calculam o estoque do cliente $i$ no período $t$ como sendo o estoque no período anterior $t$ - 1 , acrescida a quantidade recebida $q_{i k}^{t}$, subtraída a demanda $d_{i}^{t}$ no período, mais o estoque mínimo $m C_{i}$. Em (5), o estoque do fornecedor é definido. As inequações em (6) asseguram que a quantidade transportada por um veículo $k$ não exceda sua capacidade de carregamento. Em (7), garante-se que cada cliente $i$ seja visitado por apenas um veículo $k$. Os sete conjuntos de restrições seguintes modelam os requisitos para a não ocorrência de subciclos: em (8) e (9), o somatório de arcos que chegam e que saem a um determinado cliente $i$ devem ser únicos. Em (10) certifica-se que a quantidade de arcos que chegam ao depósito deve ser igual à quantidade que dele sai. Em (11) é assegurado que existam no máximo $K$ subrotas. Já o conjunto de equações em (12) garante a continuidade da rota sempre que o veículo passar por um ponto diferente do depósito. Em (13), o fluxo de produtos que passa por um nó $i$, com exceção do depósito, subtraído do fluxo que sai desse mesmo nó, resulta na quantidade abastecida para o i-ésimo cliente. Já as inequações em (14) limitam o fluxo que passa por um arco utilizado no percurso à capacidade do veículo. O conjunto de expressões (15) e (16) delimita o domínio das variáveis.

A política $O U$ é considerada em [16]-[18] e sua formulação requer duas restrições adicionais sobre a quantidade entregue aos clientes. Estendendo o modelo proposto por [18] para uma frota com múltiplos veículos, tem-se:

$$
\begin{array}{lr}
q_{i k}^{t} \geq C_{i} y_{i k}^{t}-I_{i}^{t} & \forall k \in K, t \in T, i \in V^{\prime} \\
q_{i k}^{t} \leq C_{i} y_{i k}^{t} & \forall k \in K, t \in T, i \in V^{\prime}
\end{array}
$$

O conjunto de inequações em (17) e (18) asseguram ao cliente i um carregamento igual à capacidade máxima de estocagem, dada por $C_{i}-I_{i}^{t}$, caso ele seja visitado pelo veículo k no período t $\left(y_{i k}^{t}=1\right)$, e " 0 ", caso contrário $\left(y_{i k}^{t}=0\right)$.

\section{INDICADORES DE DESEMPENHO}

Popularmente, indicadores de desempenho em logística frequentemente se limitam ao volume transportado por distância percorrida, ou simplesmente "volume por distância". A fim de ampliar a capacidade de análise, propõe-se neste trabalho uma métrica adicional para o transporte: a taxa de ocupação média da frota. Pela influência que a variabilidade da capacidade de estocagem dos clientes exerce sobre a qualidade da política de distribuição, uma métrica de estoque também é considerada.

Classicamente, o "volume por distância" expressa a razão entre a quantidade total de produto transportado pela distância total percorrida ao longo de $T$. Em um sistema logístico em três níveis, esta métrica incorpora a política de entrega aos clientes bem como a estratégia de reposição do fornecedor. Matematicamente, o indicador é definido como segue. 


$$
Q \text { tr / Dist }=\frac{\sum_{t=1}^{p} \sum_{k=1}^{K} \sum_{i=1}^{n} q_{i k}^{t}}{2 \sum_{t=1}^{p} \lambda^{t} c_{0 n+1}+\sum_{i=0}^{n} \sum_{j=0, j \neq i}^{n} \sum_{k=1}^{K} \sum_{t=1}^{p} c_{i j} x_{i j k}^{t}}
$$

A quantidade total entregue contempla o fluxo entre o segundo e o terceiro nível da cadeia (fornecedor-cliente), enquanto a distância total percorrida leva em conta os três níveis, dado que, quanto maior o fluxo de produto entre o elo fornecedor-cliente, maior deverá ser o fluxo basefornecedor. Já a taxa de utilização da frota mensura a média de ocupação de capacidade que cada veículo parte do depósito para a realização das entregas aos clientes. O indicador é calculado pela razão entre a quantidade total carregada por um veículo $k(k=1, \ldots, K)$, dada pela soma das quantidades individuais dos clientes atendidos pela rota realizada por $k$, e a capacidade nominal de carregamento $L$. A média é calculada para a frota e posteriormente para o horizonte de planejamento, produzindo um índice global apresentado a seguir.

$$
\operatorname{Tx} F r U=\frac{1}{p K} \sum_{t=1}^{p} \sum_{k=1}^{K} \sum_{i=1}^{n}\left[\frac{q_{i k}^{t} y_{i k}^{t}}{Q}\right]
$$

A equação (26) apresenta a taxa média de ocupação dos estoques dos clientes, dada pela média de ocupação ao final de um período para o conjunto $V$ ' ao longo de $T$. Este indicador capta o efeito da quantidade transportada no nível terceiro da cadeia.

$$
\text { TxInvCli }=\frac{1}{p} \sum_{t=1}^{p} \sum_{i=1}^{n}\left[\frac{I_{i}^{t}}{C_{i}}\right]
$$

Devido ao impacto que o custo de estocagem tem na operação global de sistemas $V M I$, especialmente em situações onde o produto armazenado possui grande valor, esta métrica acaba sendo indispensável para uma melhor avaliação de desempenho das estratégias de distribuição.

\section{ESTRATÉGIA DE RESOLUÇÃO}

Dada a complexidade combinatória do PRE, o modelo PLIMB proposto na seção 2 está limitado a cenários muito reduzidos. Para tal, uma estratégia heurística em três fases é proposta. Na primeira fase o período de tempo e a quantidade a ser entregue aos clientes é determinada, enquanto na fase dois as rotas de atendimento são construídas. A terceira opera melhorias sobre a solução incumbente.

A programação inicial das entregas dos clientes ao longo de $T$ baseada no ponto de pedido. A cada período $t$, um conjunto de clientes que serão atendidos $\left(E^{t}\right)$ é construído. O cliente $i$ irá pertencer à $E^{t} \mathrm{em} t \operatorname{se} d_{i}^{t}>\left(I_{i}^{t-1}-m C_{i}\right)$. A quantidade a ser entregue a este cliente é dada conforme os critérios de cada política (45 e 46), definindo-se então uma solução inicial para a decisão de quando atender e quanto entregar aos clientes.

$$
\begin{aligned}
& \text { - Maximum Level } \\
& q_{i}^{t}=d_{i}^{t}-\left(I_{i}^{t-1}-m C_{i}\right), \forall i \in V^{\prime}, \forall t \in T \\
& \text { - Order-up-to-level } \\
& q_{i}^{t}=C_{i}-\left(I_{i}^{t-1}-m C_{i}\right), \forall i \in V^{\prime}, \forall t \in T
\end{aligned}
$$

Sequencialmente uma técnica de consolidação a priori é aplicado sobre a política $M L$. Como as rotas não foram ainda determinadas, a prioridade de antecipação é dada por uma Lista de Antecipação $(L A)$, construída a partir da maior distância média de um cliente $i$ em relação ao conjunto $V^{\prime} \cup 0$. Baseado em $(L A)$, a consolidação tenta antecipar a quantidade entregue um período $t_{l}\left(q_{i}^{t l}\right)$ para um período anterior $t_{2}\left(t_{2}<t_{1}\right)$, desde que a economia no custo de transporte pela eliminação da entrega em $t_{1}$ seja maior que o aumento no custo de estocagem $\left(t_{1}-t_{2}\right)^{*} q_{i}{ }^{t l} h_{i}$. Adicionalmente, deve existir disponibilidade no estoque do cliente e o aumento da quantidade total programada nos períodos anteriores à $t_{l}$ não pode exceder a $80 \%$ da capacidade nominal da frota $\left(K^{*} L\right)$. O processo considera a reprogramação forward $\left(t_{2}=1\right)$ e backward $\left(t_{2}=t_{1}-1\right)$ (figura 2) 

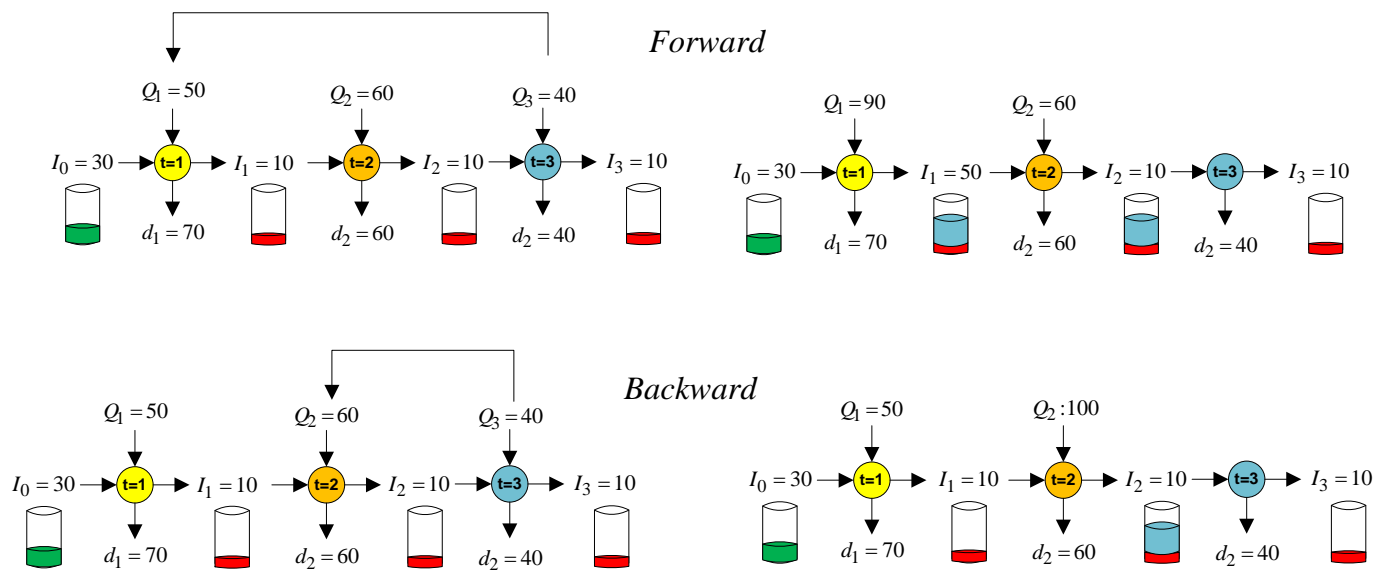

Figura 2. Antecipação das Entregas para a política $M L$

A roteirização das entregas envolve inicialmente a formação de grupos de atendimento para cada período em $T$. O agrupamento pode ser formulado como uma variante do Problema das $p$-Medianas Capacitado (PPMC) que consiste na localização de $p$-facilidades satisfazendo a demanda de um conjunto de clientes de acordo com a capacidade de atendimento de cada facilidade. Uma formulação para o problema é apresentado por [19]. Neste trabalho o PPMC é resolvido por um algoritmo de simulação direta. A partir das coordenadas de todos os elementos de $E^{t}$, onde $E^{t}=\left\{1, \ldots, n^{t}\right\}$ define o conjunto formado pelos clientes com entregas programadas no período $t$, um grid é delimitados pelas maiores e menores coordenadas $x, y$. As coordenadas para os $K$ grupos são geradas aleatoriamente, dentro dos limitantes do grid. Os clientes (elementos de $E^{t}$ ) são priorizados para designação de acordo com a diferença entre a razão da quantidade entregue em $t$ pela distância da coordenada do cliente à coordenada do grupo $k$ mais próximo, em relação à razão dessa mesma quantidade e a distância da coordenada do cliente à coordenada do segundo grupo mais próximo. Isso prioriza a designação de clientes com grandes volumes de entrega para os grupos que estão mais próximos à eles. O processo até que todos os elementos de $E^{t}$ estejam designados para todo $t, \mathrm{t}=1, \ldots, p$. Definido os grupos de atendimento, o problema original recai em até $K$ Problemas de Caixeiro Viajante (PCV) para cada período em $T$. O PCV é resolvido através das técnicas clássicas do tipo k-opt, especificamente as trocas 2-opt seguidas das trocas 3-opt. Aplicações bem sucedidas desses procedimentos no contexto do PRE podem ser verificadas em [20], [21] e [22]. Um pseudocódigo é apresentado em [23].

\subsection{REABASTECIMENTO DO FORNECEDOR}

O reabastecimento do fornecedor a partir da base é realizado na forma de uma entrega direta. Por essa estrutura, o cálculo de um lote econômico de entrega é ensejado. Seja $D F$ a demanda total do fornecedor ao longo do horizonte de planejamento. Obviamente, DF é determinada pela política de distribuição utilizada para reabastecer aos clientes. Matematicamente, o parâmetro é definido como a diferença entre tudo que o fornecedor precisa entregar ao conjunto de clientes $V^{\prime}$ em $T$, subtraído do estoque inicial do depósito. Caso o estoque inicial do fornecedor seja suficiente para atender a quantidade programada de entrega, automaticamente $D F=0$.

$$
D F=\sum_{t=1}^{p} \sum_{k=1}^{K} \sum_{i=1}^{n} q_{i k}^{t}-I_{0}^{0}
$$

O estoque médio do fornecedor $\overline{I_{0}}$ ao longo de $T$ é calculado como a média dos estoques ao final de cada período. Baseado na formulação do estoque apresentada em (5), tem-se:

$$
\overline{I_{0}}=\frac{1}{p} \sum_{t=1}^{p} I_{0}^{t}=\frac{1}{p} \sum_{t=1}^{p}\left(I_{0}^{t-1}+r^{t}-\sum_{i=1}^{n} \sum_{k=1}^{K} q_{i k}^{t}\right)=\frac{1}{p} \sum_{t=0}^{p-1} I_{0}^{t}+p^{*} r^{t}-\frac{1}{p} \sum_{t=1}^{p} \sum_{i=1}^{n} \sum_{k=1}^{K} q_{i k}^{t}
$$

Onde $r^{t}$ é a notação utilizada para a quantidade que reabastece o fornecedor no período $t$, que nesta modelagem é considerada constante ao fixo do horizonte de planejamento, sendo 
representado apenas por $r$, definindo o próprio lote de entrega. O custo de processamento do pedido de reabastecimento do fornecedor $\left(C p_{f}\right)$, é dado pela distância entre a base e o depósito multiplicada por 2 (ida e volta), definido por $C p_{f}=2 c_{0 n+1}$. A frota utilizada para essa entrega é responsabilidade da base, mas o custo de transporte é arcado pelo fornecedor. Considerando o custo de operação de um sistema de abastecimento e observando a simplificação sobre o custo de aquisição do produto, tem-se.

$$
C s_{i}=\left[\frac{1}{r} * 2 c_{0 n+1} * \sum_{t=1}^{p} \sum_{k=1}^{K} \sum_{i=1}^{n} q_{i k}^{t}-I_{0}^{0}\right]+h_{0} *\left[\frac{1}{p} \sum_{t=0}^{p-1} I_{0}^{t}+p * r-\frac{1}{p} \sum_{t=1}^{p} \sum_{i=1}^{n} \sum_{k=1}^{K} q_{i k}^{t}\right]
$$

Igualando a derivada de (31) em relação à $r^{t}$ e resolvendo para esse parâmetro, tem-se o lote econômico de reabastecimento do fornecedor conforme (27).

$$
r=\sqrt{\frac{2 c_{0 n+1} * \sum_{t=1}^{p} \sum_{k=1}^{K} \sum_{i=1}^{n} q_{i k}^{t}-I_{0}^{0}}{h_{0} * p}}
$$

O fornecedor é reabastecido de acordo com a regra do ponto de pedido, ou seja, sempre que seu estoque disponível em $t$ for menor que a quantidade total que deve ser entregue aos clientes no mesmo período.

\section{CENÁRIOS E RESULTADOS}

Os cenários gerados são baseados em [18], generalizados para uma cadeia de suprimentos em três níveis. As variáveis consideram a demanda dos clientes, níveis iniciais de estoque e localização geográfica dos vértices (clientes, fornecedor e base). O conjunto de instâncias são definidos pelos parâmetros a seguir:

- $\quad$ Número de clientes $(n): 8,10,12,16,20,25,30,35,40$ e 50 .

- Número de veículos $(K): 2$ e 3 veículos.

- Horizonte de Planejamento $(p): 5,8$ e 10 períodos.

- Distribuição da demanda do cliente a cada período $\left(d_{i}^{\dagger}\right)$ : normalmente distribuída $N\left(\mu_{i}\right.$, $\left.\sigma_{i}^{t}\right)$, baseada em uma média $\mu_{i}$ gerada a partir de uma distribuição uniforme discreta $U(10 ; 100)$, com desvio padrão por período $\sigma_{i}^{t}$ também definido por uma função de distribuição uniforme discreta limitada em $U(2 ; 10)$. A cada novo período $t$, uma nova demanda é gerada.

- Percentual Mínimo de Estocagem $\left(m C_{i}\right): \alpha C_{i}$, onde $\alpha$ é uma variável uniforme contínua entre $U[0,1 ; 0,2]$.

- Capacidade de Estocagem do Cliente $\left(C_{i}\right): g_{i} *\left(1-m C_{i}\right)^{*} \mu_{i}$, onde $g_{i}$ é um número aleatório gerado a partir de uma distribuição uniforme discreta $U(2 ; 4)$.

- Estoque Inicial no Depósito $\left(I_{0}^{0}\right): \sum_{i=1}^{p} C_{i}$

- Estoque Inicial nos Clientes $\left(I_{i}^{0}\right): C_{i}-\left(\frac{1}{p} \sum_{t=1}^{p} d_{i}^{t}\right)$

- Custo de estocagem do fornecedor $\left(h_{0}\right): 0,02$.

- Custo de estocagem do cliente $\left(h_{i}\right)$ : Baixo Custo de Estocagem: $U$ [0,02; 0,05]; Alto Custo de Estocagem: $U[0,2 ; 0,5]$.

- Capacidade do Veículo $(Q):\left(\frac{3}{2}\right) *\left(\sum_{i=1}^{n} \mu_{i}\right)$

- Distância entre os $\operatorname{arcos}(i, j)\left(c_{i j}\right): \sqrt{\left(X_{i}-X_{j}\right)^{2}+\left(Y_{i}-Y_{j}\right)^{2}}$, onde: Baixo Custo $\left(X_{i}, Y_{i}\right)$ : $U(0 ; 100)$. Coordenada da Base $U(100 ; 200)$. Médio Custo $\left(X_{i}, Y_{i}\right): U(0 ; 500)$. Coordenada da Base $U(500 ; 1000)$. Baixo Custo $\left(X_{i}, Y_{i}\right): U(0 ; 1000)$. Coordenada da Base $U(1000 ; 2000)$. 
Foram consideradas 6 configurações de custos, sendo: $L t L i$ - Baixo Custo de Transporte e Baixo Custo de Estoque; LtHi - Baixo Custo de Transporte e Alto Custo de Estoque; $\mathrm{MtLi}$ Médio Custo de Transporte e Baixo Custo de Estoque; $\mathrm{MtHi}$ - Médio Custo de Transporte e Alto Custo de Estoque; $\mathrm{HtLi}$ - Alto Custo de Transporte e Baixo Custo de Estoque; $\mathrm{HtHi}$ - Alto Custo de Transporte e Alto Custo de Estoque. Para cada uma das configurações de custo, foram geradas 10 quantidades diferentes de clientes, com 2 diferentes quantidades de veículos e 3 quantidades diferentes de períodos, totalizando $(10 * 2 * 3)=60$ instâncias. Levando em conta as 6 combinações de custos, o número de cenários gerados foi de 360 .

Os experimentos computacionais foram realizados em um processador Intel Core i3 TM de 32 bits, CPU de $3.10 \mathrm{GHz}$ com 2GB de memória, sistema operacional Windows 7 Home, Service Pack 1. A heurística proposta foi implementada em linguagem Visual Basic.NET. Foram utilizados dois critérios de parada: cada teste computacional foi limitado em 100 iterações para instâncias com até 50 clientes e 10 iterações para instâncias com 50 clientes, não podendo ultrapassar 60 minutos por teste. Cada iteração é configurada por uma nova geração aleatória de sementes durante o processo de agrupamento dos pontos de demanda. O número de iterações para a simulação do agrupamento dos pontos de demanda foi fixado em 1000.

A tabela 1 apresenta o resultado médio para cada combinação de custos considerada, com os melhores resultados destacados em negrito. É possível observar que a política $M L$ dominou os cenários em que os custos de estocagem se sobrepõe ao custo de transporte (configurações $\mathrm{Lt}$ - $\mathrm{Hi}$, $M t-H i)$. Pelo fato da política $M L$ ser flexível, a quantidade entregue varia consideravelmente a cada período. Ademais, a primeira consolidação, ainda na fase 1 , leva em conta apenas as quantidades mínimas, de acordo com a necessidade líquida de cada cliente. Já a política $O U$ é dominante para configurações com baixo custo de transporte e equivalentes entre custo de transporte e estocagem $(\mathrm{Ht}-\mathrm{Hi})$. Pela proporção entre os custos, há vantagem em priorizar estoques maiores para reduzir o número de visitas. Os tempos de processamento (coluna CPU em segundos) foram bastante competitivos tendo em vista que os valores médios não superaram 100 segundos. Pelo número menor de clientes roteirizados ao longo do horizonte de planejamento, a política $O U$ demonstrou-se mais competitiva.

Tabela 1 - Síntese dos Resultados Obtidos

\begin{tabular}{ccc|c|c|c}
\cline { 2 - 6 } & & \multicolumn{2}{c|}{ ML } & \multicolumn{2}{c}{ OU } \\
\hline TC & IC & CT & CPU & CT & CPU \\
\hline Lt & Li & 4363 & 55 & $\mathbf{4 3 5 7}$ & 40 \\
Mt & $\mathrm{Li}$ & 18253 & 74 & $\mathbf{1 7 9 0 2}$ & 42 \\
$\mathrm{Ht}$ & $\mathrm{Li}$ & 33177 & 87 & $\mathbf{3 2 1 5 3}$ & 55 \\
$\mathrm{Lt}$ & $\mathrm{Hi}$ & $\mathbf{5 9 4 4}$ & 23 & 6912 & 29 \\
$\mathrm{Mt}$ & $\mathrm{Hi}$ & $\mathbf{2 2 9 0 7}$ & 91 & 23084 & 51 \\
$\mathrm{Ht}$ & $\mathrm{Hi}$ & 37912 & 66 & $\mathbf{3 7 3 6 7}$ & 48 \\
\hline AVG & 20426 & 66 & 20296 & 44.2 \\
\hline
\end{tabular}

A tabela 2 apresenta a média dos desvios em relação aos melhores resultados obtidos para todas as instâncias. É interessante observar que embora a política $O U$ tenha dominado o desempenho médio para a configuração $L t-L i$, o desempenho da política $M L$ foi menos disperso. Isso se explica pelo fato de que, para as instâncias menores, com número de clientes inferior à 20 , o desempenho da política $M L$ foi mais estável que a política $O U$.

Tabela 2 - Desvio em relação às melhores soluções

\begin{tabular}{cc|c|c}
\hline $\mathrm{TC}$ & $\mathrm{IC}$ & ML & OU \\
\hline $\mathrm{Lt}$ & $\mathrm{Li}$ & $\mathbf{2 . 1 5 \%}$ & $2.92 \%$ \\
$\mathrm{Mt}$ & $\mathrm{Li}$ & $3.02 \%$ & $\mathbf{1 . 2 2 \%}$ \\
$\mathrm{Ht}$ & $\mathrm{Li}$ & $3.71 \%$ & $\mathbf{1 . 0 4 \%}$ \\
$\mathrm{Lt}$ & $\mathrm{Hi}$ & $\mathbf{0 . 1 2 \%}$ & $16.78 \%$ \\
$\mathrm{Mt}$ & $\mathrm{Hi}$ & $\mathbf{1 . 7 4 \%}$ & $3.26 \%$ \\
$\mathrm{Ht}$ & $\mathrm{Hi}$ & $2.83 \%$ & $\mathbf{1 . 7 8 \%}$ \\
\hline
\end{tabular}


Os resultados principais são apresentados na seção das figuras a seguir. A figura 6 apresenta o indicador $Q t r / D i s t$ para a política $M L$. No eixo horizontal está representado o custo total da instância e no eixo vertica o valor do indicador. É interessante observar que instâncias com custo total superior à 10.000 estão limitadas à uma unidade de quantidade transportada por um uma unidade de distância. Por sua vez, valores superiores à um para o indicador $Q t r / D i s t$ ocorrem apenas em instâncias com custo total inferior à 10.000, não ocorrendo nenhuma observação acima de 5 unidades de quantidade transportada por unidade de distância. Claramente há um limitador para o ganho de escala. Pode-se concluir que os resultados empíricos sugerem que cenários onde os clientes com média ou elevada dispersão geográfica (Mt e $H t)$, independentemente dos custos de estocagem envolvidos, não possibilitam ganhos de escala observado pelo supracitado indicador. Tal conclusão origina-se na tabela 1 , onde o custo total médio inferior à 10.000 está restrito à configurações apenas com reduzido custo de transporte.

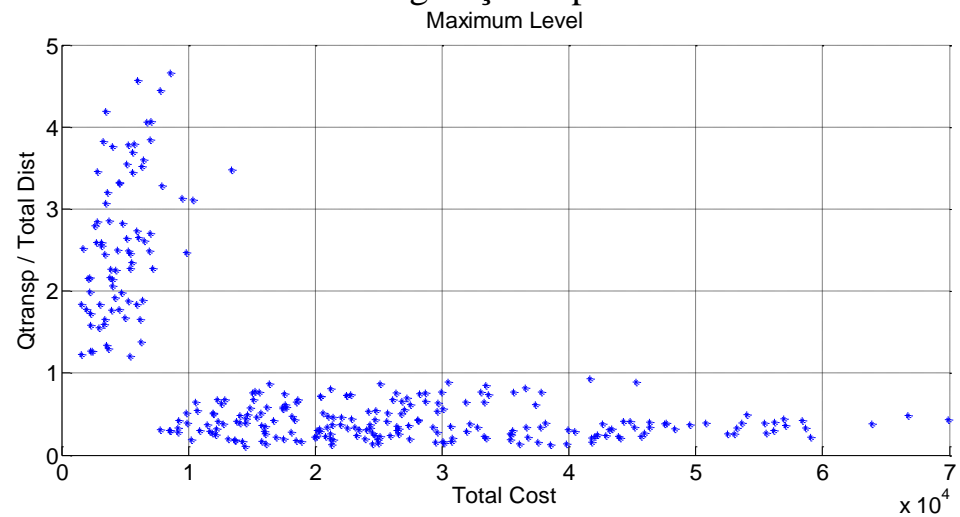

Figura 3. Qtr/Dis em relação ao custo total para a política $M L$

O indicador é apresentado para a política OU na figura 4. Há apenas algumas poucas situações em que o custo total é superior à 10.000 e quantidade transportada por distância supera a unidade. De forma geral, a mesma conclusão pode ser observada.

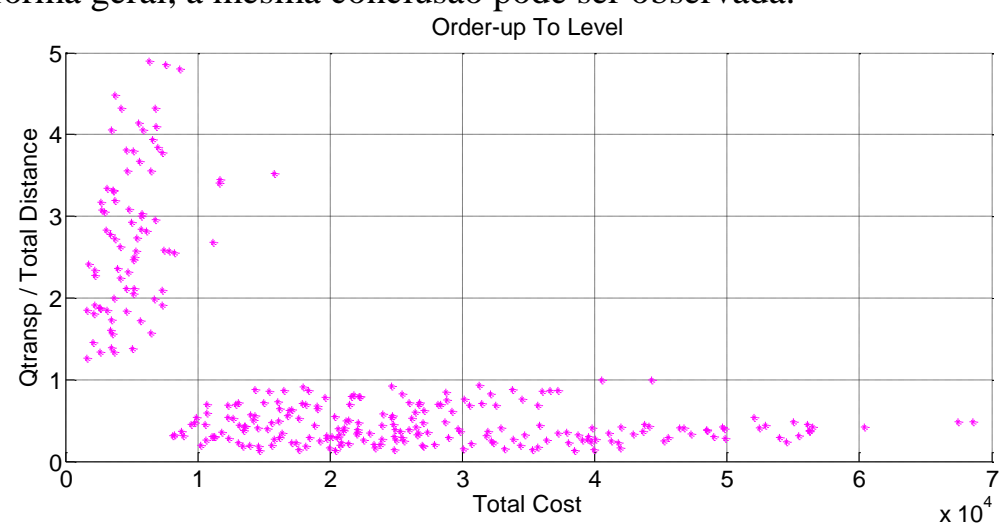

Figura 4. Qtr/Dis em relação ao custo total para a política $O U$

Com relação à taxa de uso da frota $(T x F r U)$, outro padrão se verifica na política $M L$. A frota de veículos parte do depósito com uma ocupação que varia em uma banda de $40 \%$ a $80 \%$ da capacidade de carga do veículo. Esses limites ocorrem para instâncias que demandam um custo total de até 30.000. Os cenários com alto custo de transporte $(\mathrm{Ht})$, que conforme a tabela 1 apresentam custo total superior à 30.000 , possui limitante inferior mais elevado, acima de $60 \%$ da ocupação do veículo. Pode-se concluir que os operadores de consolidação das entregas impedem que um veículo transite com baixa ocupação. 


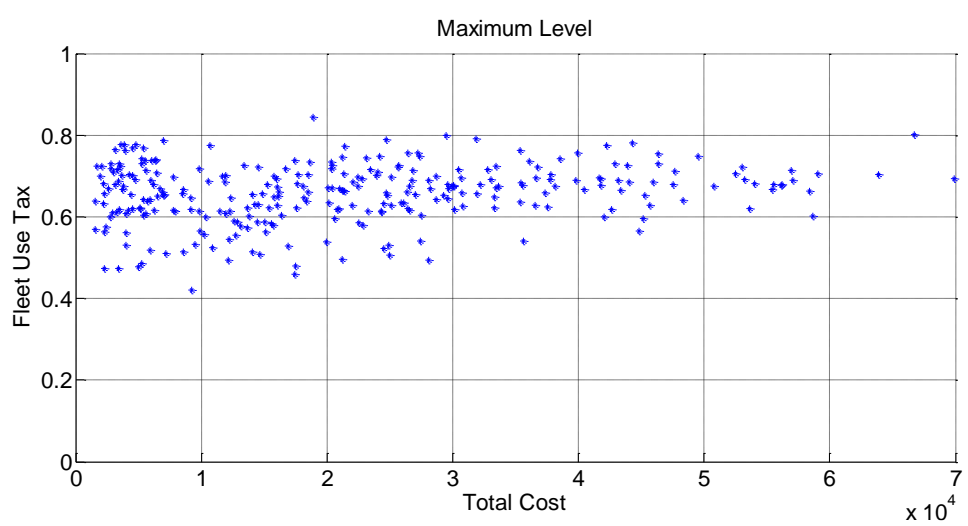

Figura 5. TxFrU em relação ao custo total para a política $M L$

A política $O U$ (figura 6) tem limites ligeiramente superiores à política $M L$, com ocupação inferior à $40 \%$ da capacidade do veículo para algumas instâncias menores. Entretanto, instâncias de maior porte (custo total igual à 40.000) também estão limitadas à variação de $60 \%$ à $80 \%$ de ocupação do veículo. Isso pode ser explicado pela estabilidade dos resultados dessas políticas apontados pela tabela 1 . Como os resultados para essas políticas foram bastante competitivos, sugere-se que instâncias de maior porte podem ter redução nos custos se as visitas forem mais frequentes, o que impacta em um volume menor carregado no veículo.

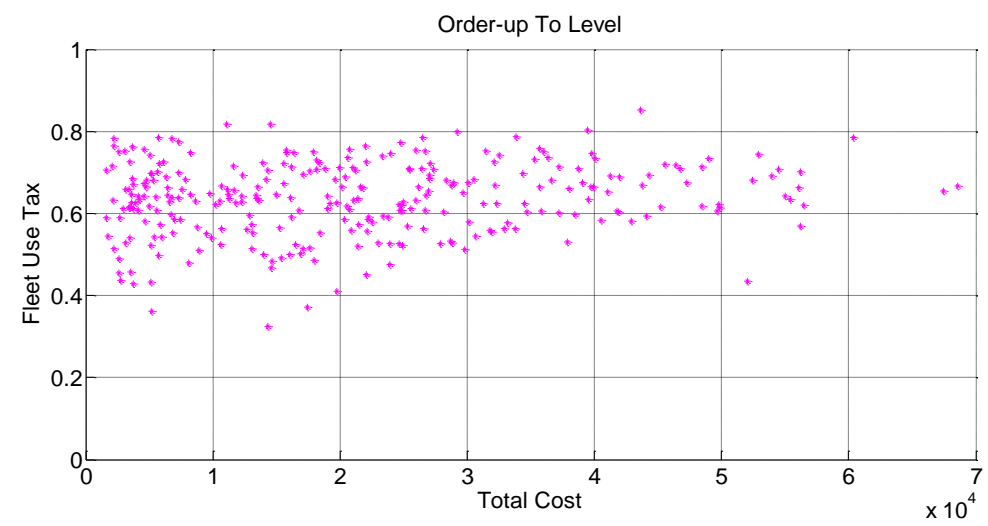

Figura 6. TxFrU em relação ao custo total para a política $O U$

Por fim avalia-se a métrica de estoque através da taxa média de estocagem dos clientes ao final dos períodos. De acordo com a estrutura da política $O U$ esperava-se que esta apresentasse maiores valores para o indicador. Entretanto, a política demonstrou-se muito estável para todas as instâncias testadas. A figura 7 mostra que a taxa média de ocupação dos estoques ao final de cada período no horizonte de planejamento ficou em $40 \%$. Devido à variabilidade na geração das instâncias, pode-se concluir que a política $O U$ não é capaz de manter os níveis de estocagem elevados nos clientes, na medida em que a frequência das entregas se reduz pelo fato do cliente ser abastecido até a capacidade máxima sempre que é visitado. Dessa maneira, para se ter maiores níveis de estoques, é necessário elevar a frequência das visitas. 


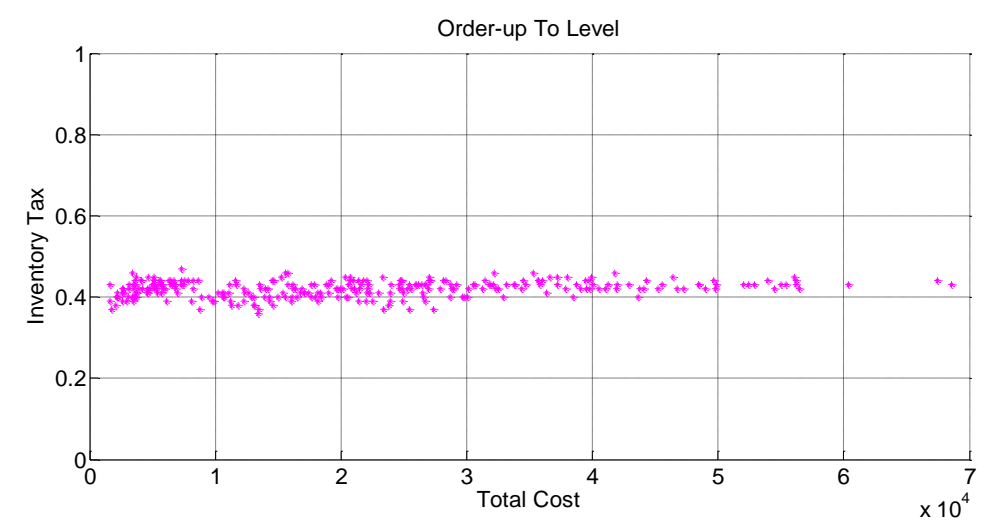

Figura 7. TxInvCli para política $O U$

Entretanto, a política ML produz uma taxa de estocagem média dos clientes ao final do período levemente inferior à $40 \%$, principalmente para instâncias com custo total inferior à 30.000. Tais cenários ocorrem com maior frequencia para as configurações com médio e baixo custo de transporte. Configurações com alto custo de transporte em geral também resultam em taxas médias de estocagem dos cliente na ordem de $40 \%$.

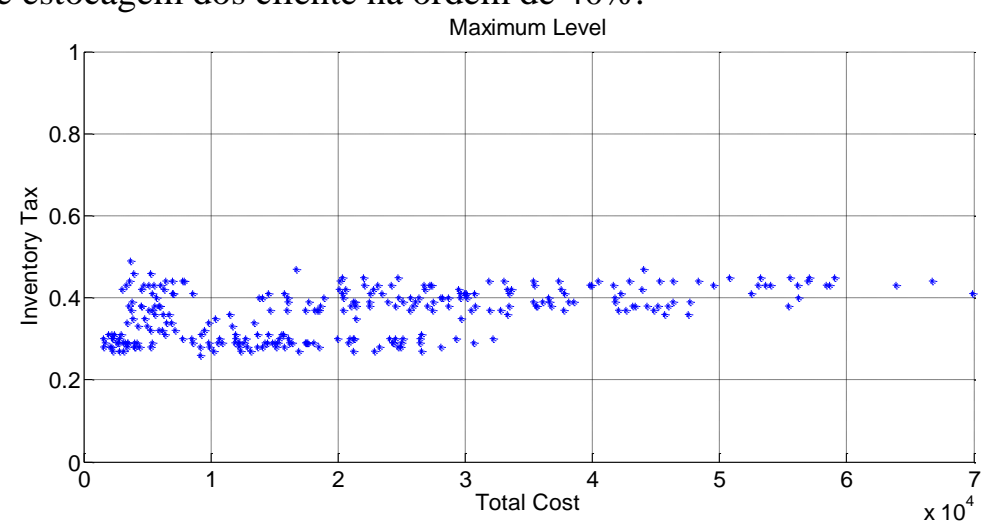

Figura 8. TxInvCli para política $M L$

\section{CONCLUSÕES}

Este estudo avaliou o desempenho logístico para problemas de roteirização com estoque gerenciado pelo fornecedor em uma cadeia de suprimentos de três níveis e demanda estocástica dos clientes. O tema possui grande pertinência em razão da vasta aplicação que o IRP possui no setor industrial e empresarial, e ainda carecia de uma discussão mais aprofundada. A heurística proposta para a resolução do problema se mostrou eficiente ao produzir resultados competitivos eem tempos de processamento relativamente baixos. As políticas de distribuição formuladas sustentam a qualidade dos resultados, ainda que não haja benchmark para uma comparação mais precisa.

Os indicadores propostos para análise de desmepenho logístico produziram resultados interessantes, e sugeriram uma direção oposta à conclusões usuais sobre a característica de cada política e a conceito de ganho de escala. Foi verificado que a partir de um determinado porte, não há mais ganho de escala pelo incremento do volume transportado pela quantidade percorrida pela frota de veículos. Além disso a, há uma certa estabilidade quanto à taxa de ocupação da frota e a taxa de esstocagem dos clientes. Entende-se que dessa forma o trabalho contribui para o aprofundamento de pesquisas sobre desempenho em cadeia de suprimentos, apontanto direcionamentos que até então não eram conhecidos.

\section{REFERÊNCIAS BIBLIOGRÁFICAS}

[1] L. C. Coelho, J. Cordeau, and G. Laporte, "Thirty Years of Inventory Routing," Transp. Sci., no. November, pp. 1-19, 2013.

[2] L. Coelho, J.-F. Cordeau, and G. Laporte, "The inventory-routing problem with transshipment," Comput. Oper. Res., vol. 39, no. 11, pp. 2537-2548, 2012. 
[3] S.-C. Liu and W.-T. Lee, "A heuristic method for the inventory routing problem with time windows," Expert Syst. Appl., vol. 38, no. 10, pp. 13223-13231, Sep. 2011.

[4] L. Coelho, J. Cordeau, and G. Laporte, "Consistency in multi-vehicle inventory-routing," Transp. Res. Part C ..., vol. 24, pp. 270-287, 2012.

[5] J. G. Rakke, M. Stålhane, C. R. Moe, M. Christiansen, H. Andersson, K. Fagerholt, and I. Norstad, "A rolling horizon heuristic for creating a liquefied natural gas annual delivery program," Transp. Res. Part C Emerg. Technol., vol. 19, no. 5, pp. 896-911, Aug. 2011.

[6] R. Gronhaug, M. Christiansen, G. Desaulniers, and J. Desrosiers, "A Branch-and-Price Method for a Liquefied Natural Gas Inventory Routing Problem," Transp. Sci., vol. 44, no. 3, pp. 400-415, Mar. 2010.

[7] E. E. Halvorsen-weare, K. Fagerholt, and M. Rönnqvist, "Vessel routing and scheduling under uncertainty in the liquefied natural gas business," Comput. Ind. Eng., vol. 64, no. 1, pp. 290-301, 2013.

[8] V. Hemmelmayr, K. F. Doerner, R. F. Hartl, and M. W. P. Savelsbergh, "Delivery strategies for blood products supplies," OR Spectr., vol. 31, no. 4, pp. 707-725, Mar. 2008.

[9] M. Christiansen, K. Fagerholt, T. Flatberg, Ø. Haugen, O. Kloster, and E. H. Lund, "Maritime inventory routing with multiple products: A case study from the cement industry," Eur. J. Oper. Res., vol. 208, no. 1, pp. 86-94, Jan. 2011.

[10] J. Alegre, M. Laguna, and J. Pacheco, "Optimizing the periodic pick-up of raw materials for a manufacturer of auto parts," Eur. J. Oper. Res., vol. 179, no. 3, pp. 736-746, Jun. 2007.

[11] J. Stacey, M. Natarajarathinam, and C. Sox, "The storage constrained, inbound inventory routing problem," Int. J. Phys. Distrib. Logist. Manag., vol. 37, no. 6, pp. 484-500, 2007.

[12] Q.-H. Zhao, S. Chen, and C.-X. Zang, "Model and algorithm for inventory/routing decision in a three-echelon logistics system," Eur. J. Oper. Res., vol. 191, no. 3, pp. 623-635, Dec. 2008.

[13] J. Li, F. Chu, and H. Chen, "A solution approach to the inventory routing problem in a three-level distribution system," Eur. J. Oper. Res., vol. 210, no. 3, pp. 736-744, May 2011.

[14] J.-H. Song and M. Savelsbergh, "Performance Measurement for Inventory Routing," Transp. Sci., vol. 41, no. 1, pp. 44-54, Feb. 2007.

[15] J. Li, H. Chen, and F. Chu, "Performance evaluation of distribution strategies for the inventory routing problem,” Eur. J. Oper. Res., vol. 202, no. 2, pp. 412-419, Apr. 2010.

[16] L. Bertazzi, G. Paletta, and M. G. Speranza, "Deterministic Order-Up-To Level Policies in an Inventory Routing Problem," Transp. Sci., vol. 36, no. 1, pp. 119-132, Feb. 2002.

[17] C. Archetti, L. Bertazzi, A. Hertz, and M. G. Speranza, "A Hybrid Heuristic for an Inventory Routing Problem," INFORMS J. Comput., vol. 24, no. 1, pp. 101-116, Feb. 2011.

[18] C. Archetti, L. Bertazzi, G. Laporte, and M. G. Speranza, "A Branch-and-Cut Algorithm for a Vendor-Managed Inventory-Routing Problem," Transp. Sci., vol. 41, no. 3, pp. 382-391, Aug. 2007.

[19] J. a. Díaz and E. Fernández, "Hybrid scatter search and path relinking for the capacitated p-median problem,” Eur. J. Oper. Res., vol. 169, no. 2, pp. 570-585, Mar. 2006.

[20] D. Popović, M. Vidović, and G. Radivojević, "Variable Neighborhood Search heuristic for the Inventory Routing Problem in fuel delivery," Expert Syst. Appl., vol. 39, no. 18, pp. 1339013398, Dec. 2012.

[21] M. Boudia and C. Prins, "A memetic algorithm with dynamic population management for an integrated production-distribution problem," Eur. J. Oper. Res., vol. 195, no. 3, pp. 703-715, Jun. 2009.

[22] N. Shukla, a. K. Choudhary, P. K. S. Prakash, K. J. Fernandes, and M. K. Tiwari, "Algorithm portfolios for logistics optimization considering stochastic demands and mobility allowance," Int. J. Prod. Econ., vol. 141, no. 1, pp. 146-166, Jan. 2013.

[23] A. A. Chaves, "Uma metaheurística híbrida com busca por agrupamentos aplicada à problemas de otimização combinatoria," Tese de Doutoramento em Computação Aplicada Instituto Nacional de Pesquisas Espaciais, 2009. 